\title{
Correlation between high frequency intravascular ultrasound and histomorphology in human coronary arteries
}

\author{
F Prati, E Arbustini, A Labellarte, B Dal Bello, L Sommariva, M T Mallus, A Pagano, \\ A Boccanelli
}

\begin{abstract}
Objective-To test the efficacy of high frequency intravascular ultrasound (IVUS) transducers in identifying lipid/necrotic pools in atherosclerotic plaques.

Methods-40 MHz transducers were used for in vitro IVUS assessment of 12 arterial segments (10 coronary and two carotid arteries, dissected from five different necropsy cases). IVUS acquisition was performed at $0.5 \mathrm{~mm} / \mathrm{s}$ after ligature of the branching points to generate a closed system. Lipid/necrotic areas were defined by IVUS as large echolucent intraplaque areas surrounded by tissue with higher echodensity. To obtain histopathological sections corresponding to IVUS cross sections, vessels were divided into consecutive $3 \mathrm{~mm}$ long segments using the most distal recorded IVUS image as the starting reference. Samples were then fixed with $10 \%$ buffered formalin, processed for histopathological study, serially cut, and stained using the Movat pentacrome method.

Results-122 sections were analysed. Lipid pools were observed by histology in 30 sections $(25 \%)$. IVUS revealed the presence of lipid pools in 19 of these sections $(16 \%$; sensitivity $65 \%$, specificity $95 \%$ ).

Conclusions-In vitro assessment of lipid/necrotic pools with high frequency transducers was achieved with good accuracy. This opens new perspectives for future IVUS characterisation of atherosclerotic plaques.

(Heart 2001;85:567-570)
\end{abstract}

Keywords: intracoronary ultrasound; atherosclerosis; plaque morphology

Coronary intravascular ultrasound (IVUS) provides quantitative information on lumen and vessel dimensions and plaque severity, as well as qualitative information on plaque composition in terms of hard and soft components and calcification. Previous IVUS studies on plaque composition, mainly performed in the early 1990 s with $20-30 \mathrm{MHz}$ transducers, showed that the technique defines calcification with high sensitivity and specificity, but is less accurate in assessing soft tissue components. ${ }^{1-9}$ Thus, although 20 and $30 \mathrm{MHz}$ transducers achieved appropriate definition of plaque morphology, the imaging of details such as the lipid pool and the fibrous cap remained poorly defined. No data are available on the characterisation of plaque morphology with high frequency transducers, which should allow more accurate definition of the soft components of the plaques.

In this study we correlated corresponding IVUS and histopathological findings in human arterial specimens obtained at necropsy from patients with atherosclerosis, to determine how accurately $40 \mathrm{MHz}$ IVUS can identify lipid/ necrotic pools.

\section{Methods}

We performed in vitro IVUS assessments, using continuous pull back, in arterial segments dissected from necropsy hearts. Arterial samples were serially sectioned in relation to IVUS markers. We then correlated the quantitative and qualitative evaluations of lipid/ necrotic pools obtained from histopathological slides with those obtained from IVUS cross sections.

\section{SAMPLE SERIES}

The pathological series comprised 12 full length arteries, 10 coronary arteries (one left main, four left anterior descending, three circumflex, and two right) and two carotid arteries dissected from five different necropsy cases (all men, age range 52-72 years). All five patients died from acute myocardial infarction.

IVUS INVESTIGATIONS

The in vitro study used an imaging catheter with a $40 \mathrm{MHz}$ mechanical transducer (Scimed, Boston Scientific Corp, Maple Grove, Minnesota, USA). The catheter transducer (diameter $0.9 \mathrm{~mm}$ ) provides high resolution cross sectional images.

Arterial segments were dissected from the epicardial fat, and all side branches and distal ends of the artery were ligated to generate a closed system. A 7 French valved sheath was fixed in the proximal end of the vessel segment by an external ring suture. The arterial segments were then suspended horizontally in a container by small metal hooks and placed in a beaker of water. Saline $(0.9 \%)$ was infused through the lateral arm of the sheath at a constant pressure $(60-80 \mathrm{~mm} \mathrm{Hg})$ using a syringomanometer. ${ }^{7}$ Internal markers (calcific deposits) and an external marker (a surgical needle) were used to confirm that ultrasound images and histological sections were aligned. 


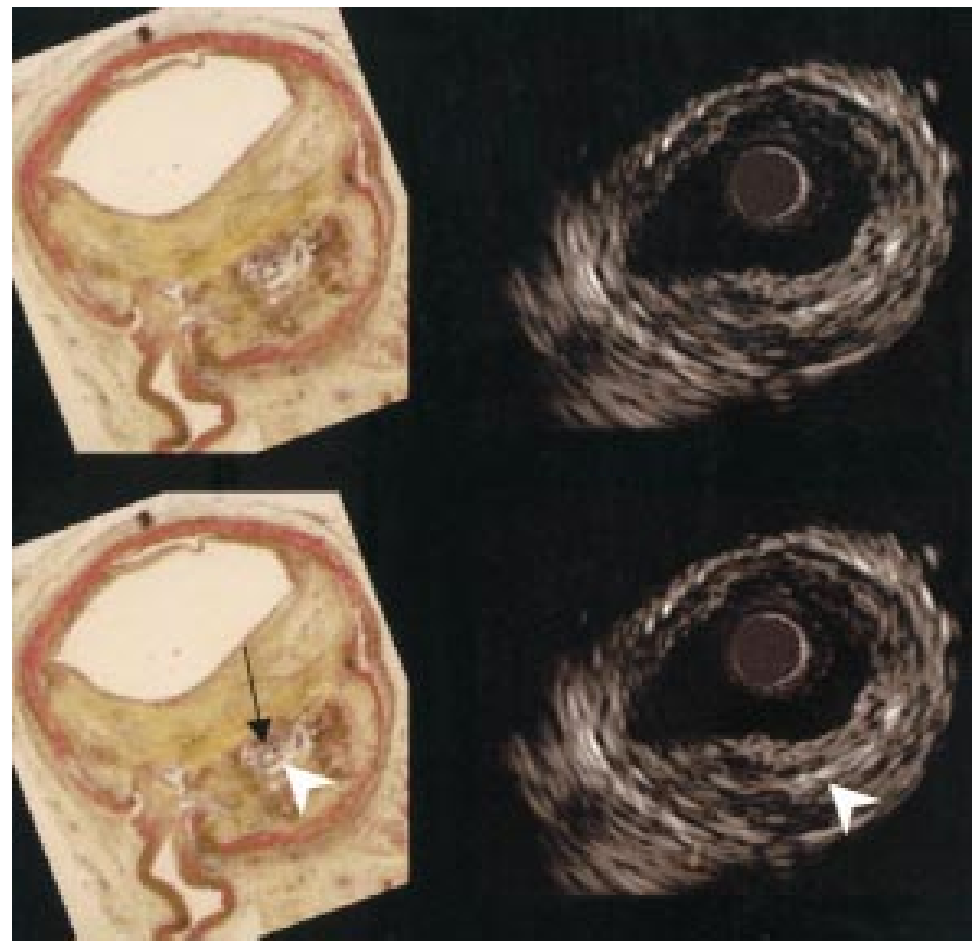

Figure 1 Photomicrograph of histological and corresponding IVUS cross sections in the left anterior descending coronary artery. Left panel: at 5 o'clock, there is a superficial calcific deposit adjacent to the emergence of a diagonal branch (black arrowhead) and a lipid pool deeply embedded in the atherosclerotic plaque (white arrowhead). Right panel: in the corresponding IVUS cross section the lipid pool cannot be identified because of echo signal attenuation caused by the presence of highly echogenic superficial fibrous tissue (arrowhead).

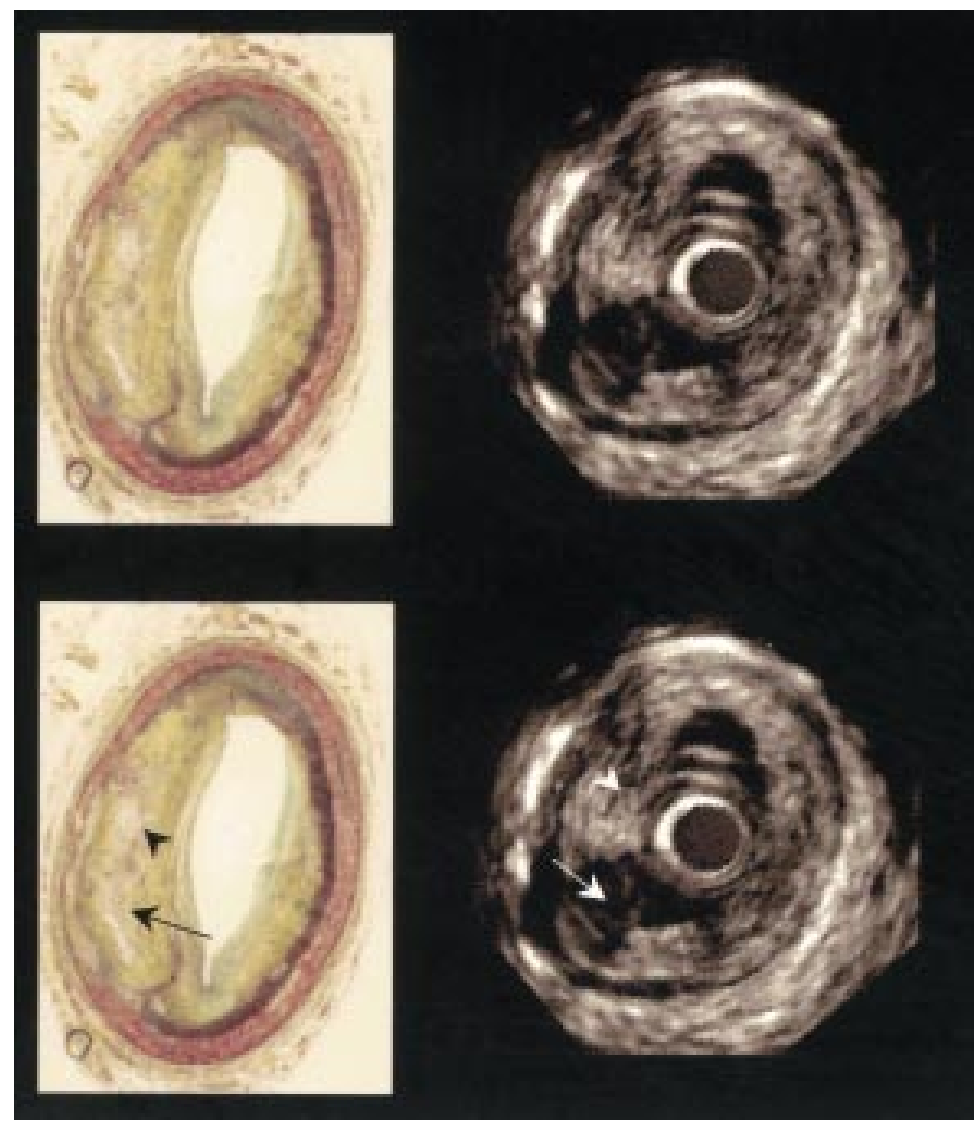

Figure 2 Photomicrograph of a histological section from the left circumflex coronary artery (left panel) and the corresponding ultrasound imaging (right panel). The histological section shows a lipid/necrotic area restricted to the 8 and 10 o'clock positions (arrows). IVUS confirms the presence of the lipid/necrotic pool at 7 o'clock, where an echolucent lake is visible (arrow). At the 7-10 o'clock position, IVUS is not capable of revealing the lipid content owing to its diffuse distribution within the atherosclerotic plaque (arrowhead).
The IVUS catheter was then inserted into the introducer and advanced within the arterial samples until the external marker was clearly imaged. The IVUS catheter was kept coaxial with respect to the arterial segments and was pulled back at a constant speed of $0.5 \mathrm{~mm} / \mathrm{s}^{10}{ }^{11}$ The first seconds of the automated pull back were discarded from analysis to avoid non-uniform movement of the catheter in the initial phase of the continuous pull back.

QUALITATIVE AND QUANTITATIVE IVUS ASSESSMENT OF LIPID/NECROTIC POOLS

Characteristics of the plaques were defined as follows:

- Lipid/necrotic areas-large echolucent areas within the plaque, circumscribed by tissue with higher echodensity.

- Fibrous tissue-plaque components having a density similar to or greater than that of the adventitia.

- Calcific deposits-highly echogenic segments having a density greater than that of the adventitia and causing acoustic shadowing.

Measurements of lipid/necrotic areas and of the thickness of the fibrous cap were obtained. The latter was defined as the minimum thickness of the tissue layer separating the lipid necrosis pool from the lumen.

\section{REPRODUCIBILITY OF OBSERVATIONS}

In each cross section, qualitative and quantitative analyses were performed by two independent observers (interobserver variability). Blinded analyses were repeated by the first observer after an interval of at least four weeks (intraobserver variability).

HISTOPATHOLOGICAL STUDY

Arterial vessels were cut into consecutive $3 \mathrm{~mm}$ long segments from distal to proximal ends using the most distal recorded IVUS image as the starting reference. A coding number was assigned to each segment. A corresponding number had been used to mark the IVUS images. Arterial samples were then fixed with $10 \%$ buffered formalin, routinely processed for histopathological study, and embedded in paraffin. Serial sections from each block were cut at $0.5 \mathrm{~mm}$ intervals.

\section{STATISTICAL ANALYSIS}

All continuous variables are expressed as mean (SD). Continuous data were compared with a paired two tailed Student's $t$ test. The results of measurements of lipid pool area and fibrous cap thickness, repeated by the first observer (intraobserver variability) and calculated by two independent observers (interobserver variability), were analysed. In order to assess intraand interobserver variability, the results were compared using a $\kappa$ test of concordance for categorical data and correlation analysis for continuous variables. ${ }^{12} \mathrm{~A}$ probability value $\mathrm{p}<0.05$ was considered significant. 


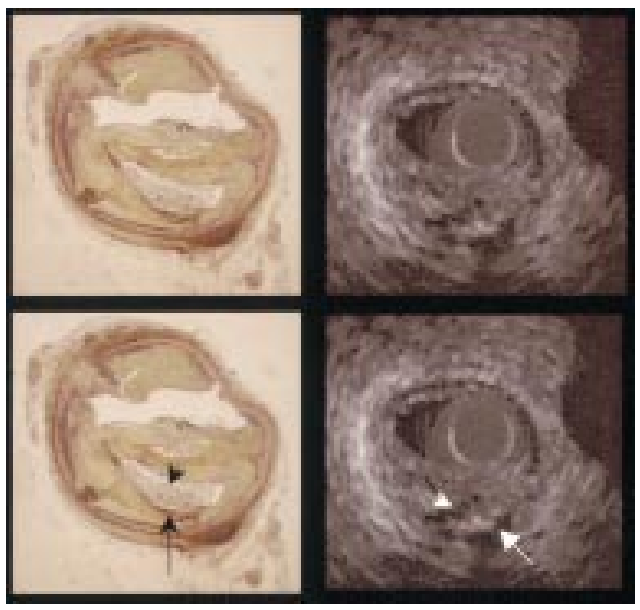

Figure 3 Photomicrograph of a histological section from the left anterior descending coronary artery (left panel) and the corresponding ultrasound imaging (right panel). A lipid/necrotic core (arrowhead), with a calcific deposit (arrow) deeply embedded in the atherosclerotic plaque, is visible at 5-6 o'clock. IVUS shows a corresponding echogenic area indicative of lipid core (arrowhead) with microcalcification (bright deposit identified by the arrow).

\section{Results}

QUALITATIVE ASSESSMENT OF PLAQUE

COMPOSITION

We analysed 122 sections. Lipid pools were observed by histology in 30 sections (25\%). IVUS revealed the presence of lipid pools in 19 sections $(16 \%)$, with a sensitivity of $65 \%$ and a specificity of $95 \%$. In five cases ( $4 \%$ ), IVUS revealed a hypoechogenic area interpreted as a lipid pool which could not be seen on histology (false positive). In all these cases the echolucency was caused by areas of shadowing from dense fibrous tissue. In 10 cases (8\%) lipid pools could not be identified by IVUS (false negative); in seven of these the misinterpretation was caused by the presence of dense fibrotic tissue and calcific deposits, leading to hypoechogenic areas that were erroneously interpreted as shadowing from these highly echogenic structures (fig 1). In the remaining three cases the misinterpretation resulted from the diffuse nature of the lipid pools, which were not localised to a definite area within the atherosclerotic plaque (fig 2).

A common observation on IVUS was the presence of small highly reflective deposits, either within or around the lipid lake; these bright deposits, corresponding to plaque microcalcifications on histology, were found in 12 of the 19 lipid pools (64\%) (fig 3).

REPRODUCIBILITY OF THE QUALITATIVE AND QUANTITATIVE ASSESSMENT OF LIPID/NECROTIC POOLS

High intraobserver agreement was obtained on necrotic lipid pool assessment (115 of 122 IVUS cross sections; $94 \%, \quad \kappa=0.63$,

Table 1 Reproducibility of the measurements

\begin{tabular}{lllll}
\hline & Analysis Ia & Analysis Ib & & Analysis II \\
\hline Area of lipid/necrotic pools $\left(\mathrm{mm}^{2}\right)$ & $0.55(0.35)$ & $0.45(0.36)$ & $\star$ & $1.00(0.48)$ \\
Thickness of fibrous cap (mm) & $0.38(0.14)$ & $0.39(0.19)$ & & $0.36(0.13)$ \\
\hline
\end{tabular}

Intraobserver variability: first (Ia) $v$ second (Ib) observation; interobserver variability: first (Ia) $v$ second (II) observer. ${ }^{\star} \mathrm{p}<0.05$. $\mathrm{p}<0.0001)$. There was also a high interobserver agreement (113 of 122 IVUS cross sections; $93 \%, \kappa=0.62, \mathrm{p}<0.0001)$.

Fibrous plaque thickness and lipid pool areas, as measured by the first and second observers, are reported in table 1. Lipid pool area was significantly smaller when measured by the first observer (mean (SD): 0.55 $(0.35) \mathrm{mm}^{2}$ v $1.00(0.48) \mathrm{mm}^{2}$, respectively, $\mathrm{p}<0.05)$. Low correlations were found for intraobserver and interobserver measurements of fibrous cap thickness $(r=0.29$, NS, and $r=-0.02$, NS, respectively). Low correlations were also obtained for intraobserver and interobserver measurements of lipid pool areas ( $r=0.45$, NS, and $r=0.41, \mathrm{NS}$, respectively).

\section{Discussion}

PLAQUE CHARACTERISATION

Atherosclerotic plaques can be grouped by IVUS into three principal subsets: plaques with predominantly soft, predominantly fibrous, or calcific composition. ${ }^{1-9}$ Previous studies on the correlation between IVUS and histology have shown that highly echogenic lesions are mostly formed of dense fibrous or calcific tissue, while plaques with a prevalent echolucent pattern contain larger amounts of loose fibrous or smooth muscle-rich tissue and thrombotic or necrotic elements. ${ }^{3}$ The IVUS characterisation of more specific details on plaque morphology, such as lipid pools, was rather cumbersome and led to different highly subjective interpretations. Occasionally, echolucent areas within atherosclerotic plaques could be properly identified as lipid/necrotic areas, but in other circumstances shadowing from dense fibrotic tissue was mistaken for lipid. ${ }^{5}$ In a recent consensus article on behalf of the European study group on intracoronary imaging, ${ }^{8}$ it was stressed that lipid deposits can be missed owing to the limited resolution of currently available transducers. Potkin and colleagues found that IVUS was quite inaccurate in identifying quadrants mainly composed of lipid: $22 \%$ of areas diagnosed by IVUS as lipid were identified by histology as fibrous plaque with echo dropout. ${ }^{6}$

The current study was designed to determine whether upgraded $(40 \mathrm{MHz})$ IVUS transducers can reliably evaluate lipid/necrotic pools within the atherosclerotic plaques. We showed that such transducers can define lipid/ necrotic pools with a sensitivity of $65 \%$ and a specificity of $95 \%$. In our study small calcific deposits, appearing as highly echogenic spots, were often observed by IVUS among the lipid lakes. This observation corresponded with the histological finding of scattered deposits of microcalcification among the lipid pools. In a previous study, based on $30 \mathrm{MHz}$ transducers, the IVUS sensitivity for microcalcification was only $64 \%$, although high specificity was preserved. ${ }^{13}$ The identification of small calcific deposits within the lipid pools using high frequency transducers is therefore an additional marker that enhances the accuracy of IVUS in identifying lipid pools. 
CLINICAL IMPLICATIONS

The possibility of characterising atherosclerotic plaques more accurately is of paramount importance. The role of the lipid content of the plaque in the pathogenensis of acute coronary events has not been fully elucidated. In current hypotheses, a large lipid core is one of the markers that define vulnerable plaques-that is, plaques that are prone to rupture and further thrombosis. ${ }^{14}{ }^{15}$ However, recent studies have shown that vessel thrombosis also occurs in "fibrous" plaques without a lipid core, and in atherosclerotic plaques with thick caps. In these lesions plaque erosion rather than rupture is the local substrate for the thrombosis that causes acute coronary syndromes. ${ }^{16}{ }^{17}$ Thus better definition of plaque components and lipid necrosis pools may be keys to providing more reliable markers for future studies on the fate of vulnerable plaques. Possibly the in vivo characterisation of plaques with a large lipid core, and their distinction from plaques with prevalent fibrous/ proliferative composition, may contribute to identifying potentially vulnerable plaques. Furthermore, proper characterisation of plaque composition in serial IVUS studies intervals could be of help in clinical studies on the effects of drug treatment on plaque size, composition, and possible regression.

TECHNICAL LIMITATIONS

Although our study shows that $40 \mathrm{MHz}$ transducers can identify necrotic lipid pools accurately, there are still some shortcomings in the definition of plaque component. Based on our results, qualitative assessment of lipid pools can be misdiagnosed in the presence of highly echogenic tissue or where the lipid content is distributed diffusely within the atherosclerotic plaque. In the former, highly echogenic tissue-such as calcific or dense fibrous tissue - impairs the identification of lipid pools because acoustic shadowing produces an area of low echogenicity. In the latter, the irregular diffuse distribution hampers the assessment of lipid pools, which can be better visualised if they are well circumscribed in a definite region of the atherosclerotic plaque.

The possibility of visualising and measuring structural details such as lipid/necrotic areas and the thickness of the fibrous cap could provide valuable additional information for the in vivo identification of vulnerable lesions. With this in mind, we attempted to measure the lipid/necrotic pool area and the fibrous cap thickness, but we were unable to obtain reliable measurements in either case, the results being poorly reproducible, both within and between observers.

Further improvements in IVUS technology are required to overcome these limitations and to provide additional morphological information, such as precise measurements of core and fibrous cap thickness. Potential options include IVUS transducers with frequencies higher than $40 \mathrm{MHz}$, digital processing of IVUS images, and radiofrequency analysis of backscatter signals. $^{5}$

A possible limitation of our study is that our information was derived from an in vitro system and therefore plaque assessment might have been facilitated by the absence of backscatter from blood cells. However, previous in vivo studies on plaque characterisation-obtained with transducers at lower frequencies - showed that blood cells can be properly identified and separated from plaque components. ${ }^{13}$

\section{CONCLUSIONS}

In vitro assessment of lipid/necrotic pools with high frequency transducers was achieved with good accuracy. This opens new perspectives on the future IVUS characterisation of atherosclerotic plaques.

The study was supported by a grant from the Italian Foundation "Centro per la Lotta contro l'Infarto." IVUS analyses were performed at the European Imaging Laboratory, Rome, Italy.

1 Nissen SE, Gurley JC, Grines CL, et al. Intravascular ultrasound assessment of lumen size and wall morphology in normal subjects and patients with coronary artery disease. Circulation 1991;84:1087-99.

2 Gussenhoven EJ, Essed CE, Lancee CT, et al. Arterial wall characteristic determined by intravascular ultrasound imaging. An in vitro study. 7 Am Coll Cardiol 1989;14:94752 .

3 Rasheed Q, Nair R, Sheehan H, et al. Correlation of intracoronary ultrasound plaque characteristics in atheroscleotic coronary artery disease patients with clinical variables. Am f Cardiol 1994;73:753-8.

4 Siegel RJ, Ariani M, Fishbein MC, et al. Histopathologic validation of angioscopy and intravascular ultrasound. Circulation 1991;84:109-17.

5 Metz JA, Yock PG, Fitzgerald PJ. Intravascular ultrasound: basic interpretation. Cardiol Clin 1997;15:1-15.

6 Potkin BN, Bartorelli AL, Gessert JM, et al. Coronary artery imaging with intravascular high frequency ultrasound. Circulation 1990;81:1575-85.

7 Nishimura RA, Edwards W, Warnes CA, et al. Intravascular ultrasound imaging: in vitro validation and pathologic correlation. $\mathcal{F}$ Am Coll Cardiol 1990;16:145-54.

8 Di Mario C, Gorge G, Peters R, et al, on behalf of the study group on intracoronary imaging of the working group of coronary circulation of the European Society of Cardiology. Clinical application and image interpretation in intraogy. Clinical application and image interpretation in
coronary ultrasound. Eur Heart f 1998;19:207-29.

9 Palmer ND, Northridge D, Lessells A. In-vitro analysis of coronary atheromatous lesions by intravascular ultrasound. Reproducibility and histological correlation of lesion morphology. Eur Heart F 1999;20:1701-6.

10 Prati F, Di Mario C, Moussa I, et al. In-stent neointima proliferation correlates with the amount of residual plaque burden outside the stent. Circulation 1999;99:1011-14.

11 Mintz GS, Popma JJ, Pichard AD, et al. Patterns of calcifications in coronary artery disease. A statistical analysis of intravascular ultrasound and coronary angiography in 1155 lesions. Circulation 1995;91:1959-65.

12 Landis JR, Koch GG. The measurement of observer agreement for categorical data. Biometrics 1977;33:159-74.

13 Friedrich GJ, Moes NY, Mühlberger, et al. Detection of intralesional calcium by intracoronary ultrasound depends on ralesional calcium by intracoronary ultrasound depend

14 Falk E, Shah PH, Fuster V. Coronary plaque disruption. Firculation 1995;92:657-71.

15 MacIsaac AI, Thomas JD, Topol EJ. Toward the quiescent coronary plaque. F Am Coll Cardiol 1993;22:1228-41.

16 Farb A, Burke AP, Tang AL, et al. Coronary plaque erosion without rupture into a lipid core. a frequent cause of coronary thrombosis in sudden coronary death. Circulation 1996;93:1354-63.

17 Arbustini E, Burke A, Dal Bello B, et al. Plaque erosion is a major substrate for coronary thrombosis in acute myocardial infarction. Heart 1999;82:269-72. 